\title{
Social Impact Assessment of Storm Surge Disaster Through Dynamic Neural Network Model
}

\author{
Cheng Cheng ${ }^{\mathrm{a}}$, Qingtian Zeng, ${ }^{\mathrm{a}, \mathrm{b}, *}$, Hua Zhao ${ }^{\mathrm{a}}$, Wenyan Guo ${ }^{\mathrm{a}}$, and Hua Duan ${ }^{\mathrm{a}}$ \\ ${ }^{a}$ College of Computer Science and Engineering, Shandong University of Science and Technology, Qingdao, 266590, China \\ ${ }^{b}$ College of College of Electronic and Information Engineering, Shandong University of Science and Technology, Qingdao, 266590, China
}

\begin{abstract}
Storm surges are one of the most serious marine disasters in the world. Storm surge disasters bring not only sudden loss of life and property, but also a series of invisible social impacts. The social impact of storm surge disasters varies with time and is difficult to assess. In this paper, we firstly analyze the social impact index system in storm surge disasters using big data. Secondly, we extract the content of social impact factors from storm surge disasters. Finally, we use the back propagation neural network (BPNN) model to assess the level of social impact. The input of the model are factors after linear weighted fusion, and the output is the social impact assessment level of storm surge disasters. Experimental results show that our approach can accurately assess the level of social impact at different times, and the assessment model can assist disaster managers in making dynamic solving countermeasures.
\end{abstract}

Keywords: storm surge; social impact; internet big data; BPNN

(Submitted on September 15, 2019; Revised on September 28, 2019; Accepted on October 22, 2019)

(C) 2019 Totem Publisher, Inc. All rights reserved.

\section{Introduction}

Storm surge disasters are one of the most serious marine disasters in the world. They are caused by strong winds and sudden changes of atmospheric pressure accompanied by storm transits, such as tropical cyclones, extratropical cyclones, and squall lines at sea, that cause sea surface oscillation or a non-periodic abnormal rise (decrease) phenomenon superimposed on the astronomical tide level. If the storm surge overlaps with the astronomical high tide and the water level exceeds the "warning line", the water level will rise sharply. The seawater will break down the embankment, causing heavy losses to industry, agriculture, fisheries, transportation, port construction, and people's lives and property [1].

The impact of storm surge disasters has two meanings: one is the risk of storm surge disasters, and the other is the social impact caused by storm surge disasters. The correct assessment of the losses caused by storm surge disasters is key for post-disaster reconstruction. At present, many scholars have focused on direct economic losses caused by storm surge disasters [2-4] and the vulnerability assessment of hazard-affected bodies [5]. However, not enough attention has been paid to the social impact of storm surge disasters. Social impact assessment is mainly concerned with assessing the social impact of disasters [6]. A storm surge disaster will affect society in many aspects, such as human social relations, social organizational structure, social public safety, family livelihood systems, and individuals' physical and mental health. There is a lack of research on the social impact of storm surges. The effective assessment of the social impact of storm surge disasters is an important basis for governments to formulate policies for social recovery and the rehabilitation of the lives of residents in disaster-stricken areas.

In the time after a storm surge disaster, relevant data will be generated online at an alarming rate. Internet big data provides a new way for disaster management. It is used to visualize, analyze, and predict disasters. Big data is changing social assistance and crisis management [7-10]. Some scholars have combined big data with storm surge disaster prediction [11-12], while others have focused on loss assessment [13]. In order to overcome this issue, we propose a model that combines Internet big data with BPNN to assessment storm surge disasters' social impact. In this paper, we innovatively

\footnotetext{
* Corresponding author.

E-mail address: qtzeng@163.com
} 
propose Internet factors and quantify them. The social impact of storm surge disasters is assessed by collecting real-time Internet big data and the BPNN model. In the process of emergency management and decision-making of storm surge disasters, adopting effective information technology will improve the ability of rapid response to the social impact of storm surge disasters. It will minimize the impact of disasters on society, demonstrating important theoretical significance and practical value.

\section{Social Impact Assessment Framework for Storm Surge Disasters}

We propose a social impact assessment model for storm surge disasters. The overall process framework is shown in Figure 1. The paper is structured as follows. Firstly, we analyze the social impact factors of storm surge disasters. By using the available Internet big data, synthesizing expert opinions, and consulting relevant literature, a set of social influencing factors of storm surge disasters is constructed. The social impact factors of storm surge disasters are integrated with innovative factors: public concern factors and psychosocial factors. Then, factor extraction is based on multi-dimensional Internet big data. We use the social impact factor extraction of storm surge disasters based on pattern matching. Finally, the evaluation of the model is based on a big data set extracted from the Internet. The social impact of storm surges based on an artificial neural network is used in the evaluation model.

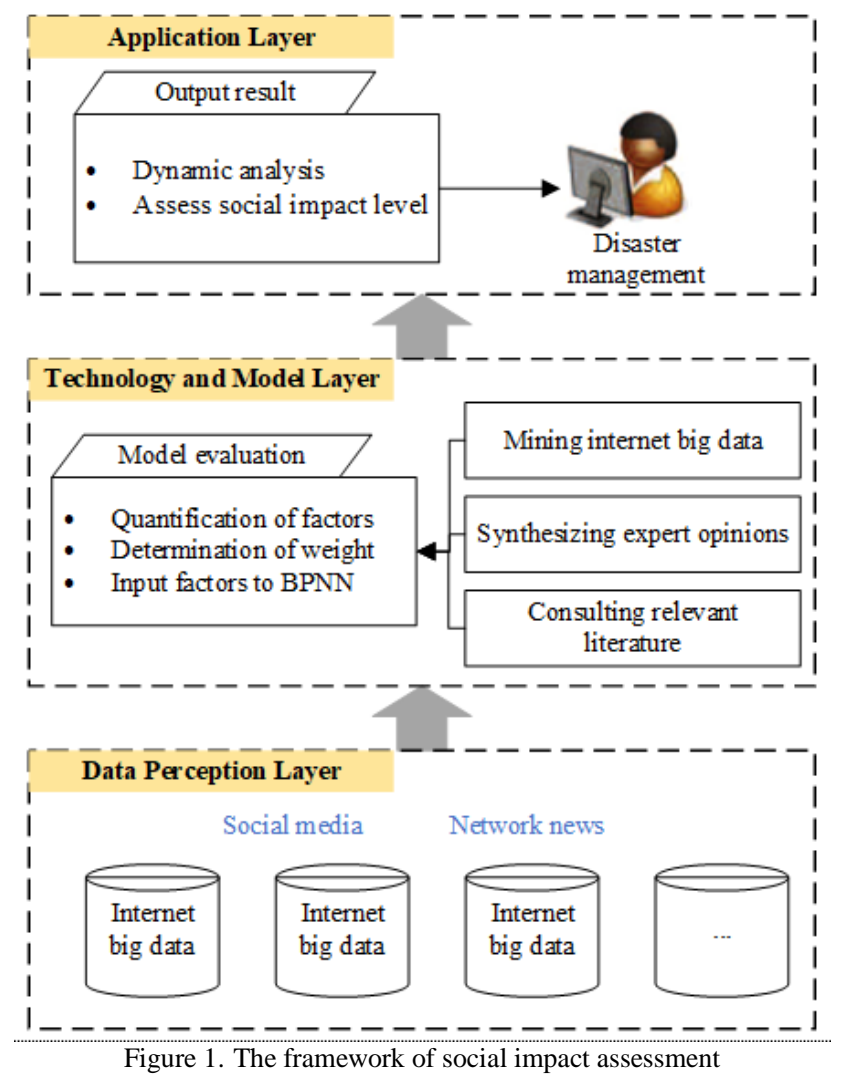

\section{Analysis of Social Impact Factors of Storm Surge Disasters}

\subsection{The Construction of Social Impact Index System of Storm Surge Disasters}

We focus on the following criteria in the analysis of social impact factors of storm surges: scientific, accurate, operational, and applicable. Based on the existing evaluation factors, this paper excavates the new dimensions and aspects of the social impact assessment of storm surge disasters. We formulate more complete and objective social impact assessment factors of storm surge disasters and determine the correlation among factors. In this paper, three methods are used to obtain the initial social impact factors of storm surge disasters: (1) consulting with experts, (2) reviewing existing literature, and (3) extracting specific social impact factors of storm surge disasters from multi-dimensional Internet big data. Through actual investigation and summary, we divide the social impact system of storm surge disasters into the following six parts: (a) personnel factors, (b) daily life factors, (c) daily livelihood factors, (d) public facilities factors, (e) public concern factors, and (f) psychosocial factors. With the increasing influence of Internet communication, people are paying more and more 
attention to storm surge disasters. For public concern factors, this paper defines the index of social concern, including the number of microblog forwarding, news reports, and the time of storm surge disasters in microblogs, which exists in social networks [14]. For psychosocial factors, emotional analysis technology is used to automatically mine for storm surge disasters opinions, views, and other information in social media. The emotional tendencies of storm surge disasters mainly include positive and negative emotions. Factors 1-4 were proposed in reference [15]. Factors 5-6 are innovative factors proposed in this paper and are unique evaluation factors in the Internet big data.

\subsection{Quantification of Factors and Determination of Weight}

Ensure the comprehensiveness of the input factors in the evaluation neural network model. From the aspect of information collection, the more factors considered, the more important information lost in the social factors of storm surge disasters that can be avoided. In order to accurately assess the social impact of storm surge disasters, it is necessary to extract the main content from all the impact factors. We determine the index system layer by layer. The structure of the social impact factor index system of storm surge disasters is shown in Figure 2.

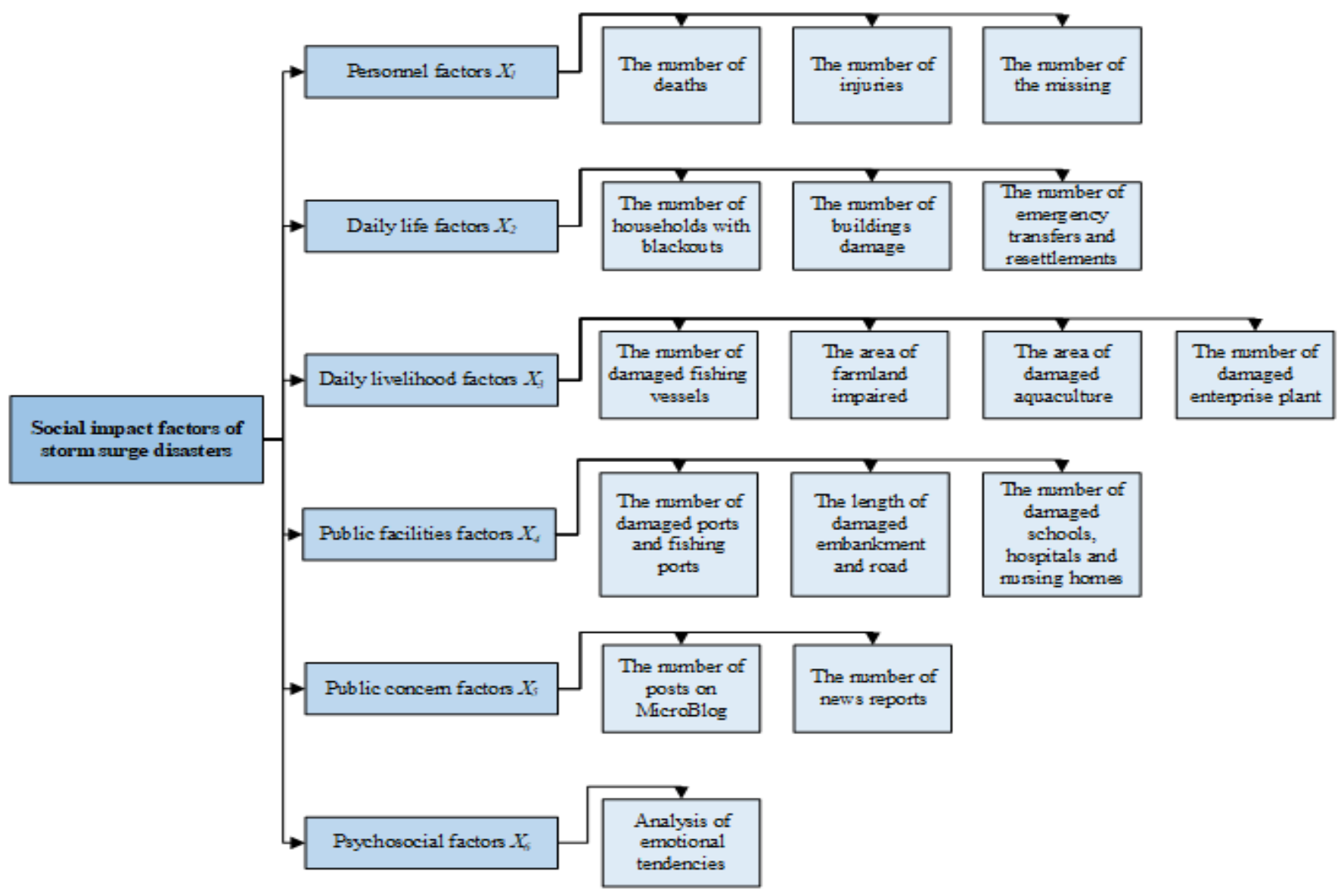

Figure 2. The construction of the social impact index system of storm surge disasters

The index system constructed in this paper adopts a combination of the analytic hierarchy process and the expert scoring method. After repeated debugging, we determine each factor and its weight. The factor weights are consistent with the storm surge disaster reality, and they are designed according to the requirements of the overall storm surge disaster social impact assessment model. The comprehensive evaluation of social impact factors of storm surge disasters includes six first-level factors and 16 second-level factors. We determine the corresponding weight of each factor. The factor and their weights are summarized in Table 1.

After obtaining the extracted factor data, it is necessary to quantify the social impact factor data of storm surge disasters. These factors are mostly numerical attributes, such as the number of deaths and the number of news reports. We disregard non-numerical psychosocial impact factors that use a corresponding numerical model, such as negative emotions corresponding to 0 and positive emotions corresponding to 1 . 
Table 1. Weight of social impact factors of storm surge disasters

\begin{tabular}{|c|c|c|c|c|}
\hline \multirow{17}{*}{$\begin{array}{l}\text { Social impact } \\
\text { factors of } \\
\text { storm surge } \\
\text { disasters }\end{array}$} & First level index $\left(W_{i}\right)$ & Weight $\left(S_{i}\right)$ & Second level index $\left(W_{j}\right)$ & Weight $\left(S_{j}\right)$ \\
\hline & \multirow{3}{*}{ Personnel factors $\left(X_{1}\right)$} & \multirow{3}{*}{0.3} & The number of deaths & 0.5 \\
\hline & & & The number of injuries & 0.3 \\
\hline & & & The number of the missing & 0.2 \\
\hline & \multirow{3}{*}{ Daily life factors $\left(X_{2}\right)$} & \multirow{3}{*}{0.2} & The number of households with blackouts & 0.4 \\
\hline & & & The number of buildings damage & 0.4 \\
\hline & & & The number of emergency transfers and resettlements & 0.2 \\
\hline & \multirow{4}{*}{ Daily livelihood factors $\left(X_{3}\right)$} & \multirow{4}{*}{0.2} & The number of damaged fishing vessels & 0.2 \\
\hline & & & The area of farmland impaired & 0.2 \\
\hline & & & The area of damaged aquaculture & 0.2 \\
\hline & & & The number of damaged enterprise plant & 0.2 \\
\hline & \multirow{3}{*}{ Public facilities factors $\left(X_{4}\right)$} & \multirow{3}{*}{0.1} & The number of damaged ports and fishing ports & 0.4 \\
\hline & & & The length of damaged embankment and road & 0.3 \\
\hline & & & $\begin{array}{c}\text { The number of damaged schools, hospitals and } \\
\text { nursing homes }\end{array}$ & 0.3 \\
\hline & \multirow{2}{*}{ Public concern factors $\left(X_{5}\right)$} & \multirow{2}{*}{0.1} & The number of posts on microblog & 0.5 \\
\hline & & & The number of news reports & 0.5 \\
\hline & Psychosocial factors $\left(X_{6}\right)$ & \multicolumn{3}{|c|}{0.1} \\
\hline
\end{tabular}

\section{Extraction of Social Impact Factors of Storm Surge Disasters based on Internet Big Data}

After a storm disaster, a large amount of data is generated on the Internet. These unstructured text data in news and social media contain useful information. When a storm surge disaster occurs, related departments release and update information related to the disaster in a timely manner, and the general public expresses their opinions and views on social platforms. The information is widely disseminated and diffused through social media, forming large storm surge disaster Internet data. The paper is based on Python language, and we use the scrapy framework to collect data from Sina Microblog and Sina News.

The extraction of social impact factors of storm surge disasters involves extracting data and emotional information related to the social impact of storm surge disasters from social media texts. We analyze the time distribution characteristics of factors and evaluate the impact level of storm surge disasters according to the extracted factors. There are two main ways to extract the social impact factors of conventional storm surge disasters: pattern matching and machine learning. The method based on pattern matching refers to extracting event information under the guidance of some patterns, while patterns refers to abstract representations of information to be extracted. It embodies the components of specific information, which can be set or acquired manually or automatically. The method based on pattern matching involves extracting event information under the guidance of some patterns. By analyzing the presentation of social impact factors of storm surge disasters in large Internet data, we find that the description of these factors in the text has a relatively fixed format. Then, we choose the method based on pattern matching in this paper to extract the social impact factors of storm surge disasters.

\subsection{Traditional Factors Extraction}

The method based on pattern matching mainly involves regular expressions, which are special strings composed of ordinary characters and special characters. It can be interpreted into multiple strings according to specific grammar rules and matched to the target strings.

The personnel factors in storm surge disasters mainly include death and injury. The regular expressions are in the following two forms:

(1) (Measure Word) (Number) (The Description Personnel Loss Description)

(2) (Number) (The Description Personnel Loss Description)

The Description Personnel Loss Description: Killed | Dead | Loss | Death | Missing | Injury …

Measure Word: At least | About | Near | More | Most $\cdots$

Number: Arabic Number | English Number $\cdots$

Social impact indicators are dynamic, and they change with the occurrence of disasters. The time dimension reflects the change of social impact of disasters, so it is necessary to extract the update time of disaster impact factors from the social Internet corpus. The three commonly used date representation formats and their corresponding regular expressions are as follows: 
(1) dd day of mm month in yyyy year: ([0-9]+)[lu5e74]([0-9]+)[lu6708]([0-9]+)[lu65e5]

(2) dd-mm-yyyy: $([0-9]+)[-]([0-9]+)[-]([0-9]+)$

(3) dd.mm.yyyy: ([0-9]+)[^.]([0-9]+)[\.]([0-9]+)

Other factors for storm surge disasters are similar to the personnel factors, such as the number of emergency transfers and resettlements, the number of households with blackouts, the number of house damages, and the number of factory buildings damaged.

Relevant data acquired by web crawlers usually need to divide the text into sentences for subsequent processing, which is usually based on the end mark, exclamation mark, and question mark. In order to improve efficiency, it is necessary to filter out irrelevant sentences, such as sentences containing only one or two words. The extraction process includes two main parts: the extraction of factor statistical times and the extraction of factor values. The algorithm process is as follows:

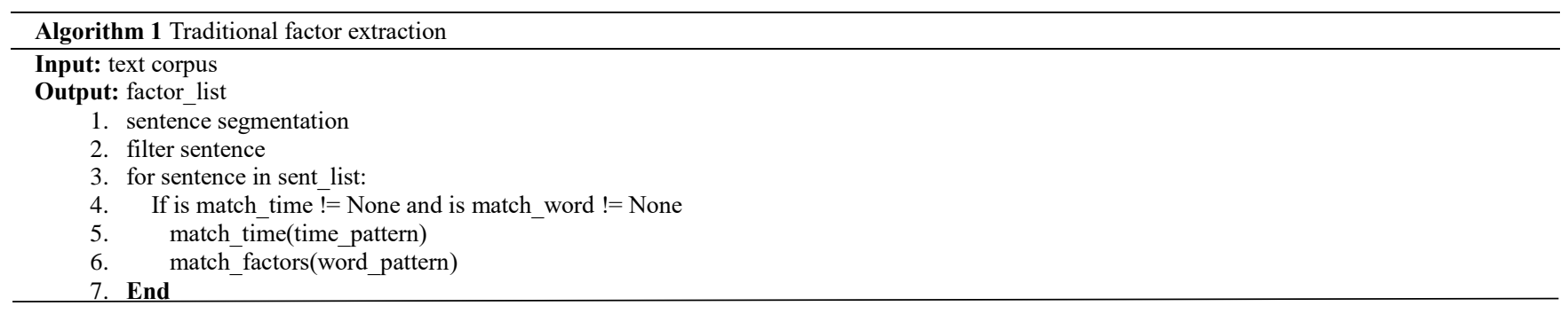

\subsection{Network Indicators Extraction}

In this paper, the proposed factors of social concern and psychosocial impact are unique factors for Internet big data. The social attention index is defined as the sum of the number of microblogs and news extracted from the Internet. The number of microblogs, reviewers, and forwarders that contain the content of a topic are often used to measure the heat of a topic. Generally speaking, the more microblogs there are, the hotter the topic is. In this paper, we collect the data generated within five days after a typhoon storm surge, use keywords to query the content of microblogs, capture the data of microblog posts, and collect and count the total number of related microblogs. We define it as the index of social concern.

Psychosocial impact factors are used to mine the views and attitudes of the general public in commentary information. In this paper, they are defined as the overall emotional analysis of the content of the blog post crawling storm surge disaster events, and it is judged whether the emotional content is negative or positive. The collected blog data of typhoon storm surge are collected, and the emotional value of the blog is calculated by using NLPIR (an emotional analysis tool) [16]. Full-text emotional discrimination is the automatic recognition of emotional words and the automatic calculation of their weights. Using a co-occurrence relationship and the Bootstrapping strategy, the iteration is repeated to generate new emotional words and weights.

\section{Social Impact Assessment Model of Storm Surge Disaster based on Artificial Neural Network}

The neural network model I-J-P network is shown in Figure 3. It is a BPNN model with two hidden layers. The first hidden layer is any neuron in $I$ expressed by $i$. The second hidden layer is any neuron in $J$ represented by $j$. The output layer is $P$, in which neurons are represented by $p . w_{m i}$ is the network weights of the input layer and the first hidden layer. $w_{i j}$ is expressed as the network weights of the first hidden layer and the second hidden layer. $w_{j p}$ represents the network weights of the second hidden layer and the output layer. $\theta_{i}$ is the threshold of the first hidden layer node. $\theta_{j}$ is the threshold of the second hidden layer node. $\theta_{p}$ represents the threshold of the output layer node.

The BP neural network needs to be adjusted by calculating the weights and thresholds of the network input layer nodes, output layer node, and hidden layer node. The transfer function in this network model I-J-P network uses a sigmoid function, $f(x)=\frac{1}{1+e^{-\alpha x}}$. Input a sample $X_{k}=\left[x_{k 1}, x_{k 2}, \cdots, x_{k m}\right], k=(1,2, \cdots, N)$. For the output layer, the actual input value is $Y_{k}=$ $\left[y_{k p}\right]^{\mathrm{T}}$, and the ideal output value is $d_{k}=\left[d_{k p}\right]^{\mathrm{T}}$. In the BP neural network, the error signal is transmitted from the back to the front. We need to modify the weights of the connections layer by layer. The weight value revision between the $J-P$ layers is $w_{j p}(n+1)=w_{j p}(n)+\Delta w_{j p}(n)$. The weight value revision of the $I-J$ layer is $w_{i j}(n+1)=w_{i j}(n)+\Delta w_{i j}(n)$. The threshold of hidden nodes in layer $I$ is $\theta_{i}(n+1)=\theta_{i}(n)+\Delta \theta_{i}(n)$. The threshold value of hidden nodes in layer $J$ is $\theta_{j}(n+1)=\theta_{j}(n)+\Delta \theta_{j}(n)$. The threshold value of output nodes in the $P$ layer is $\theta_{p}(n+1)=\theta_{p}(n)+\Delta \theta_{p}(n)$. When the 
total error energy $E(n)=\frac{1}{2} \sum_{p=1}^{p} e_{k p}^{2}(n)<\epsilon$ stops, the error between the output result and the expected set value reaches the set value.

According to the above analysis, the model of the artificial neural network chart for the social impact assessment of storm surge disasters is defined as shown in Figure 3. In the process of forward propagation, the input sample data is computed through the hidden layer nodes layer by layer, and the output results are finally obtained in the output layer. The error is obtained by comparing the output result with the expected set value. The error is modified by the output layer through the back propagation of the network connection to modify the connection weights and thresholds, so that the error of network learning is continuously reduced.

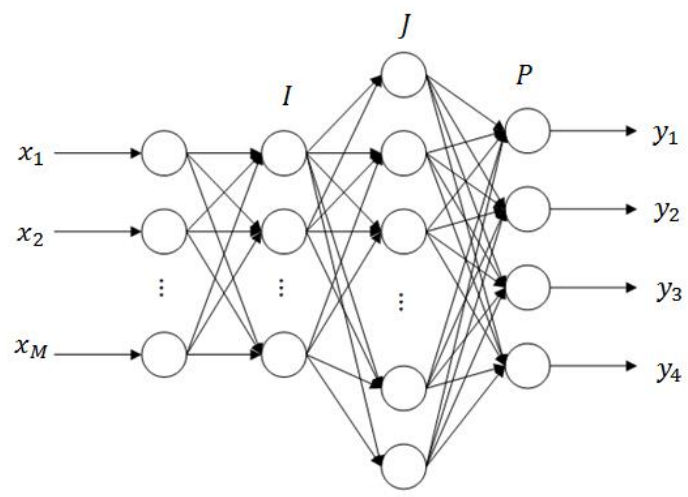

Figure 3. The neural network model

Based on the results of large data mining and evaluation factors, we established a universal evaluation model for storm surge disaster evaluation. Linear weighting is applied to each factor, and the optimized factors is input into the neural network to construct a more comprehensive social impact assessment system for storm surge disasters. Many factors are numerical attributes, and some are non-numerical attributes. In order to eliminate the impact of different orders of magnitude and dimensions, it is necessary to standardize the original data. Quantitative analysis of each factor is carried out accurately to obtain the value of each factors. Then, factors are fused to determine the relationship of each evaluation factor, and the weight of factors is determined by linear weighting. Finally, the weighted factor information is input to the neural network.

\section{A Case Study}

In this paper, we collect Internet big data of 79 storm surges in coastal China in the past five years, recorded in China's Marine Disaster Bulletin from 2013 to 2017. Temperature storm surges exist in the Internet data and have a weak effect on society. The experimental data are based on 67 typhoon surges in the past five years, from 2013 to 2017.

We analyze the case of the Typhoon Hato storm surge disaster in Zhuhai on August 22, 2017. Typhoon Hato was the thirteenth named storm in the Pacific in 2017. At 14:00 on August 20, 2017, it formed over the Northwest Pacific Ocean. After that, the intensity strengthened continuously. On August 22, it became a strong tropical storm at 8:00 and a typhoon at 15:00. At 7:00 on August 23, it became a strong typhoon, jumping two levels in a day, the strongest of which was $15(48 \mathrm{~m} / \mathrm{s}$, revised to 16 in the yearbook, 52m/s [2]). It landed in Zhuhai City, Guangdong Province at around 12:50 as a strong typhoon $(14 \mathrm{~m} / \mathrm{s}, 45 \mathrm{~m} / \mathrm{s})$. On August 24 , it weakened to a tropical depression at 14:00 and stopped numbering at 17:00 by the Central Meteorological Observatory.

Firstly, the social impact factors of storm surges related to casualties are extracted. Among 485 news reports on Typhoon Hato, 224 were related to human losses. Through the extraction method, the result of personnel loss is shown in Figure 4 as follows.

The other extraction results of the disaster are shown in Figure 5, including the number of households with blackouts, the number of damaged buildings, the number of emergency transfers and resettlements, the number of damaged fishing vessels, the area of farmland impaired, the area of damaged aquaculture, the number of damaged enterprise plants, the number of damaged ports and fishing ports, the length of damaged embankments and roads, and the number of damaged schools, hospitals, and nursing homes. 


\begin{tabular}{|lll|}
\hline 23 August,2017 & $17: 26$ & three people died and more than 20 were slightly injured \\
24 August,2017 & $15: 07$ & 16 people were killed \\
24 August,2017 & $23: 41$ & 9 people died \\
25 August,2017 & $09: 18$ & killed 17 people \\
25 August,2017 & $16: 00$ & 11 people died, 1 people missing \\
25 August,2017 & $16: 58$ & killed 12 people \\
25 August,2017 & $20: 37$ & killed 17 people \\
\hline
\end{tabular}

Figure 4. The extraction results of personnel factors of the Typhoon Hato storm surge disaster

\begin{tabular}{|c|c|c|}
\hline \multirow[t]{3}{*}{24 August, 2017} & $23: 41$ & 6425 houses collapsed \\
\hline & & 75 million mu of crops were affected \\
\hline & & 611,000 households were cut off due to disasters \\
\hline \multirow[t]{4}{*}{25 August, 2017} & $16: 00$ & more than 6600 houses collapsed \\
\hline & & more than 800 were damaged to varying degrees \\
\hline & & 68.2 thousand hectares of crops were affected \\
\hline & & 2.7 million electricity users were affected \\
\hline
\end{tabular}

Figure 5. The extraction results of some factors of the Typhoon Hato storm surge disaster

The social impact assessment model of storm surge disasters is constructed as a four-layer BP neural network. We set the input layer to six neurons, the hidden layer to eight and ten neurons, and the output layer to four neurons. The learning rate is 0.5 , the momentum factor is 0.9 , the target error is 0.01 , and the number of iterations is 300 . We use the sigmoid function and linear function in the hidden layer of the neural network. BPNN is a non-linear system. The setting of initial weights can be considered the most important part of the structure of the neural network. Each neural network needs to select appropriate initial weights and thresholds. In general, the initial weights and thresholds are randomly selected between $(-1,1)$.

In this paper, 67 typhoon storm surges from 2013 to 2017 are used. The data set of the first 66 disasters are divided into 330 optimized training groups according to the day. Five sets of data for different time periods of the Typhoon Hato storm surge disaster are used as test data: 24:00 on August 23, 24:00 on August 24, 24:00 on August 25, 24:00 on August 26, and 24:00 on August 27. Finally, the evaluation results show that the storm surge event of 24:00 on August 23 was Grade III, and the level was upgraded to Grade II after the event of 24:00 on August 24. Table 2 shows the evaluation results of the BPNN model. This method has a certain reference value for guiding practical works.

Table 2. The assessment results of BPNN model

\begin{tabular}{|c|c|c|c|c|c|}
\hline \multirow{2}{*}{ Event number } & \multicolumn{3}{|c|}{ Disaster classification } & Assessment \\
level
\end{tabular}

\section{Conclusions}

Based on the characteristics of storm surge disasters, this paper evaluates the social influencing factors of storm surge disasters and establishes an index system. In order to accurately mine the social impact factors of storm surge disasters, we established an index system of social impact factors based on storm surge disasters and excavated potential and valuable factor information of storm surge disasters based on large data of a multi-dimensional network. Then, we evaluated the social impact factors of storm surge disasters and used linear weighting to fuse the various factors in the neural network. Finally, the main factors extracted from multi-dimensional large data were simulated in a BP neural network to evaluate the social impact factors of storm surge disasters. Empirical analysis showed that the training sample results of the linear 
weighted BP neural network evaluation model constructed in this paper have efficient generalization ability and evaluation accuracy, and they can be used to analyze and evaluate the social impact of storm surge disasters. It was also shown that the evaluation model has strong reliability and operability. When storm surge disasters occur, the social impact of storm surge disasters can be assessed in time, and the evaluation grade information of social impact can be obtained. This will greatly improve the timeliness of disaster relief, help dispatch materials and personnel for storm surge disaster relief, and reduce the losses of storm surge disasters and the impact of storm surge disasters on society.

In our work, we only used typhoon storm surge disasters. Temperate storm surge disasters were not included in the experimental data, and we only studied the overall social impact of typhoon storm surges, which are more destructive. In the experiment, the time dimension of data partition was divided into 24 hours. In the later stage, more fine-grained time division experiments can be carried out.

\section{Acknowledgements}

This work was supported in part by the NSFC (No. 61472229, 61602279, 71704096, and 31671588), the Science \& Technology Development Fund of Shandong Province of China (No. 2016ZDJS02A11, ZR2017BF015, and ZR2017MF027), the Humanities and Social Science Research Project of the Ministry of Education (No. 16YJCZH154, 16YJCZH041 16YJCZH012, and 18YJAZH017), the Taishan Scholar Climbing Program of Shandong Province, and the SDUST Research Fund (No. 2015TDJH102).

\section{References}

1. X. Jin, X. Shi, J. Gao, T. Xu, and K .Yin, "Evaluation of Loss Due to Storm Surge Disasters in China based on Econometric Model Groups," International Journal of Environmental Research and Public Health, Vol. 15, No. 4, pp. 604, 2018

2. K. Yin, W. Qian, and X. Li, "The Evaluation Techniques of the Socio-Economic Loss Caused by Storm Surge Disaster," Marine Environmental Science, Vol. 31, No. 6, pp. 835-837+842, 2012

3. M. Zhu, Z. Sun, L. Tang, and L. Yu, "Study on Basic Framework of Disaster Social Impact Assessment," Journal of Natural Disasters, Vol. 24, No. 4, pp. 7-14, 2015

4. J. Zhang, "A Vulnerability Assessment of Storm Surge in Guangdong Province, China," Human and Ecological Risk Assessment: An International Journal, Vol. 15, No. 4, pp. 18, 2009

5. G. Elisabetta and P. Valentin, "Storm Surge Disaster Risk Management: The Xynthia Case Study in France," Journal of Risk Research, Vol. 16, No. 7, pp. 825-841, 2013

6. Q. Liu and S. Yang, "Review of Different Methodologies and Risk Assessment Models in Storm Surge Disaster Management," Applied Mechanics and Materials, No. 580-583, pp. 2628-2634, 2014

7. S. Akte and S. F. Wamba, "Big Data and Disaster Management: A Systematic Review and Agenda for Future Research," Annals of Operations Research, Vol. 9, pp. 1-21, 2017

8. A. Crowe, "The Social Media Manifesto: A Comprehensive Review of the Impact of Social Media on Emergency Management," Journal of Business Continuity and Emergency Planning, Vol. 5, No. 1, pp. 409, 2011

9. Q. Shi, H. Wang, D. Li, X. Shi, C. Ye, and H. Gao, "Maximal Influence Spread for Social Network based on MapReduce," in Proceedings of ICYCSEE 2015, pp. 128-136, 2015

10. D. Pohl, A. Bouchachia, and H. Hellwagner, "Online Indexing and Clustering of Social Media Data for Emergency Management," Neurocomputing, Vol. 172, No. C, pp. 168-179, 2015

11. M. Bajo and G. Umgiesser, "Storm Surge Forecast Through a Combination of Dynamic and Neural Network Models," Ocean Modelling, Vol. 33, No. 1, pp. 1-9, 2010

12. S. Yang, X. Liu, and Q. Liu, "A Storm Surge Projection and Disaster Risk Assessment Model for China Coastal Areas," Natural Hazards, Vol. 84, No. 1, pp. 1-19, 2016

13. Q. Feng and Q. Liu, "Pre-Assessment for the Loss Caused by Storm Surge based on the SVM-BP Neural Network," Marine Environmental Science, Vol. 36, No. 4, pp. 615-621, 2017

14. Z. He, Z. Cai, Q. Han, W. Tong, L. Sun, and D. Li, "An Energy Efficient Privacy-Preserving Content Sharing Scheme in Mobile Social Networks," Personal and Ubiquitous Computing, Vol. 20, No. 5, pp. 833-846, 2016

15. L. Tan, K. Chen, J. Wang, and L. Yu, "Assessment on Storm Surge Vulnerability of Coastal Regions During the Past Twenty Years," Scientia Geographica Sinica, Vol. 31, No. 9, pp. 1111-1117, 2011

16. "NLPIR-ICTCLAS, Natural Language Processing and Information Retrieval Sharing Platform Retrieved," (http://ictclas.nlpir. org/, last accessed on March 08, 2019)

Cheng Cheng is currently pursuing her Ph.D. at Shandong University of Science and Technology. Her research interests include machine learning and emergency management.

Qingtian Zeng received his Ph.D. in computer software and theory from the Institute of Computing Technology at the Chinese Academy of Sciences in 2005. He was a visiting professor at City University of Hong Kong in 2008 . He is 
currently a professor at Shandong University of Science and Technology. His current research interests include Petri nets, process mining, and knowledge management.

Hua Zhao received her B.S. degree in computer science and technology from Liaocheng University in 2001. She received her M.S. degree and Ph.D. in computer application technology from Harbin Institute of Technology (HIT) in 2003 and 2008, respectively. She currently is an associate professor in the College of Computer Science and Engineering at Shandong University of Science and Technology. Her research interests include processing mining and text mining.

Wenyan Guo received her M.S. degree in computer technology from Qingdao University in 2016. She is currently pursuing her Ph.D. at Shandong University of Science and Technology. Her current research interests include process mining and business process management.

Hua Duan received her B.S. degree and M.S. degree in applied mathematics from Shandong University of Science and Technology in 1999 and 2002, respectively. She received her Ph.D. in applied mathematics from Shanghai Jiaotong University in 2008. She is an associate professor at Shandong University of Science and Technology. Her research interests include Petri nets, business process management, and machine learning. 